Lei Cao, Ariana Hall, and Selcuk Koyuncu*

\title{
A short note on extreme points of certain polytopes
}

https://doi.org/10.1515/spma-2020-0005

Received August 29, 2019; accepted December 30, 2019

Abstract: We give a short proof of Mirsky's result regarding the extreme points of the convex polytope of doubly substochastic matrices via Birkhoff's Theorem and the doubly stochastic completion of doubly substochastic matrices. In addition, we give an alternative proof of the extreme points of the convex polytopes of symmetric doubly substochastic matrices via its corresponding loopy graphs.

Keywords: Doubly (sub)stochastic matrices; Symmetric doubly (sub)stochastic matrices, Extreme points.

MSC: $15 \mathrm{~A} 51,15 \mathrm{~A} 83$

\section{Introduction}

An $n \times n$ nonnegative matrix is called a doubly (sub) stochastic matrix if the sum of each row and each column is (less than or) equal to 1 . The convex sets of all $n \times n$ doubly stochastic matrices and doubly substochastic matrices are denoted by $\Omega_{n}$ and $\omega_{n}$, respectively. Both $\Omega_{n}$ and $\omega_{n}$ have been studied intensively in [4]. A matrix $A$ is an extreme point of a convex set $S$ if every convex decomposition of the form

$$
A=\lambda A_{1}+(1-\lambda) A_{2},(0 \leq \lambda \leq 1)
$$

where $A_{1}$ and $A_{2}$ in $S$ implies that $A_{1}=A_{2}=A$.

We let $\Omega_{n}^{t}=\left\{A \in \Omega_{n} \mid A=A^{t}\right\}$ denote the set of all $n \times n$ symmetric doubly stochastic matrices and let $\omega_{n}^{t}=\left\{B \in \omega_{n} \mid B=B^{t}\right\}$ denote the set of all $n \times n$ symmetric doubly substochastic matrices.

A well known result of Birkhoff [1] characterized all extreme points of $\Omega_{n}$, stated as the following theorem.

Theorem 1.1. ([1]) $P_{n}$ is an extreme point of $\Omega_{n}$ if and only if $P$ is an $n \times n$ permutation matrix.

A square $(0,1)$-matrix is called a subpermutation matrix or a partial permutation matrix if it has at most one 1 in each row and each column. The extreme points of $\omega_{n}$ were characterized by Mirsky [6] in the following theorem.

Theorem 1.2. ([6]) $Q_{n}$ is an extreme point of $\omega_{n}$ if and only if $Q$ is an $n \times n$ subpermutation matrix.

In Section 2, we provide an alternative proof of Theorem 2.2. In Section 3, we give equivalent characterizations of the extreme points of $\Omega_{n}^{t}$ and $\omega_{n}^{t}$ in the language of graph theory and a concise proof of the extreme points of $\omega_{n}^{t}$.

Lei Cao: Department of Mathematics, Nova Southeastern University, E-mail: lcao@nova.edu Ariana Hall: Department of Mathematics, University of North Georgia, E-mail: AMHALL4323@ung.edu ${ }^{\star}$ Corresponding Author: Selcuk Koyuncu: Department of Mathematics, University of North Georgia, E-mail: skoyuncu@ung.edu 


\section{Classical Case}

Given an $n \times n$ doubly substochastic matrix $B_{n}=\left(b_{i j}\right)_{i, j=1}^{n}$, there always exists an $m \times m$ doubly stochastic completion matrix $A_{m}$ such that $B_{n}$ is an $n \times n$ submatrix of $A_{m}$, where $n \leq m$. Indeed, denote $r_{i}$ the $i$ th row sum of $B_{n}$ and $c_{j}$ the $j$ th column sum of $B_{n}$, then

$$
\left(\begin{array}{ll}
B_{n} & D_{n} \\
E_{n} & B_{n}^{t}
\end{array}\right)
$$

is a $2 n \times 2 n$ doubly stochastic matrix where $D_{n}$ is the diagonal matrix with diagonal entries $1-r_{1}, 1-r_{2}, \ldots, 1-$ $r_{n}$ and $E_{n}$ is the diagonal matrix with diagonal entries $1-c_{1}, 1-c_{2}, \ldots, 1-c_{n}$. In [3], the authors give the lower bound of $m$ and provide methods to construct $A_{m}$ when $m$ minimal. The results in [3] imply the following proposition.

Proposition 2.1. Let $B_{n}$ be an $n \times n$ doubly substochastic matrix, then there always exists an $m \times m$ doubly stochastic matrix $A_{m}$ such that $B_{n}$ is the $n \times n$ principal submatrix on the upper left comer of $A_{m}$.

We are now in the position to provide an alternate proof of Theorem 1.2.

Theorem 2.2. ([6]) Let $n$ be a positive integer. $Q$ is an extreme point of $\omega_{n}$ if and only if $Q$ is an $n \times n$ subpermutation matrix.

Proof. On the one hand, it is clear that all $n \times n$ subpermutation matrices are extreme points of $\omega_{n}$.

On the other hand, let $B_{n} \in \omega_{n}$. According to Proposition 2.1, there exists

$$
A_{n+k}=\left(\begin{array}{cc}
B_{n} & C_{k} \\
R_{k} & M_{k}
\end{array}\right) \in \Omega_{n+k}
$$

where $C_{k}$ is an $n \times k$ matrix, $R_{k}$ is a $k \times n$ matrix and $M_{k}$ is a $k \times k$ matrix. Applying Theorem 1.1, there exists $(n+k) \times(n+k)$ permutation matrices $P_{1}, P_{2}, \ldots, P_{t}$ such that

$$
A_{n+k}=\sum_{i=1}^{t} c_{i} P_{i}
$$

where $c_{i}>0$ and $\sum c_{i}=1$. Truncate the last $k$ rows and $k$ columns of each matrix in (1), we have

$$
B_{n}=\sum_{i=1}^{t} c_{i} Q_{i}
$$

where $Q_{i}$ are subpermutation matrices. Since $B_{n}$ is arbitrary, any $n \times n$ doubly substochastic matrix can be written as a convex combination of subpermutation matrices, meaning that there are no more extreme points besides subpermutation matrices.

\section{Symmetric Case}

The extreme points of the set $\Omega_{n}^{t}$, all symmetric $n \times n$ doubly stochastic matrices, were characterized by Katz ([5], also see [2]) which can be generalized as the following theorem.

Theorem 3.1. ([5], Lemma 1 in [2]) Let $P$ be an $n \times n$ permutation matrix. Then $\frac{1}{2}\left(P+P^{t}\right)$ is an extreme point of $\Omega_{n}^{t}$ if and only if $P$ does not contain any even cycle longer than 2 . 
For an $n \times n$ symmetric matrix $A$, we associate a loopy graph in the following way to represent the structure of the non-zeros of $A$. Let $V=\left\{v_{1}, v_{2}, \cdots, v_{n}\right\}$ be the set of vertices. The loopy graph associated with $A$ is the graph $G(A)$ with vertex set $V$ such that there is an edge connecting $v_{i}$ and $v_{j}$ if and only if $a_{i, j} \neq 0$.

Let $P$ be an $n \times n$ permutation matrix. Then there is a one to one correspondence between cycles longer than 2 contained in $P$ and cycles in the loopy graph associated with $\frac{1}{2}\left(P+P^{t}\right)$. Indeed, if $\sigma=\left(i_{1}, i_{2}, \ldots, i_{k}\right)$ is a cycle contained in $P$ longer than 2, then there is a cycle

$$
v_{i_{1}} \rightarrow v_{i_{2}} \rightarrow \ldots \rightarrow v_{i_{k}} \rightarrow v_{i_{1}}
$$

contained in the associated loopy graph of $\frac{1}{2}\left(P+P^{t}\right)$ and vice versa. Hence, Theorem 3.1 in [5] can be stated as the following.

Corollary 3.2. Let $P$ be an $n \times n$ permutation matrix. Then $\frac{1}{2}\left(P+P^{t}\right)$ is an extreme point of $\Omega_{n}^{t}$ if and only if the loopy graph associated with $\frac{1}{2}\left(P+P^{t}\right)$ does not contain even cycles longer than 2 .

Proposition 3.3. Any $n \times n$ symmetric doubly substochastic matrix A can be written as a convex combination of the matrices of the form $\frac{1}{2}\left(Q+Q^{t}\right)$ where $Q$ is an $n \times n$ subpermutation matrix.

The proof of Proposition 3.3 is essentially the same as the proof of Theorem 9 in [2]. Before we give the following lemma, we would like to mention that we do not consider loops to be cycles, and since loopy graphs are undirected, the shortest possible cycle is on three vertices.

Lemma 3.4. Let $Q$ be an $n \times n$ subpermutation matrix. Then $\frac{1}{2}\left(Q+Q^{t}\right)$ is not an extreme point of $\omega_{n}^{t}$ if the loopy graph associated with $\frac{1}{2}\left(Q+Q^{t}\right)$ contains at least one even cycle.

Proof. Denote the loopy graph associated with $\frac{1}{2}\left(Q+Q^{t}\right)$ by $G$. Suppose $G$ contains an even cycle $C_{2 k}$

$$
v_{i_{1}} \rightarrow v_{i_{2}} \rightarrow \ldots \rightarrow v_{i_{2 k}} \rightarrow v_{i_{1}}
$$

where $k \geq 2$. Denote the submatrix of $\frac{1}{2}\left(Q+Q^{t}\right)$ corresponding to $\mathcal{C}_{2 k}$ by $A_{2 k}$. We construct the matrix $A_{1}$ by putting 1 in the places

$$
\left(i_{1}, i_{2}\right),\left(i_{2}, i_{1}\right),\left(i_{3}, i_{4}\right),\left(i_{4}, i_{3}\right), \ldots,\left(i_{2 k-1}, i_{2 k}\right),\left(i_{2 k}, i_{2 k-1}\right)
$$

and the matrix $A_{2}$ by putting 1 in the places

$$
\left(i_{2}, i_{3}\right),\left(i_{3}, i_{2}\right),\left(i_{4}, i_{5}\right),\left(i_{5}, i_{4}\right), \ldots,\left(i_{2 k}, i_{1}\right),\left(i_{1}, i_{2 k}\right) .
$$

Note that both $A_{1}$ and $A_{2}$ are symmetric permutation matrices and

$$
A_{2 k}=\frac{1}{2} A_{1}+\frac{1}{2} A_{2}
$$

and hence $\frac{1}{2}\left(Q+Q^{t}\right)$ is not an extreme point.

Lemma 3.5. Let $Q$ be an $n \times n$ subpermutation matrix. Then $\frac{1}{2}\left(Q+Q^{t}\right)$ is not an extreme point of $\omega_{n}^{t}$ if the loopy graph associated with $\frac{1}{2}\left(Q+Q^{t}\right)$ contains a path longer than 1 .

Proof. Denote the loopy graph associated with $\frac{1}{2}\left(Q+Q^{t}\right)$ by $G$. Suppose $G$ contains a path $\mathcal{P}_{k}$

$$
v_{i_{1}} \rightarrow v_{i_{2}} \rightarrow \ldots \rightarrow v_{i_{k}}
$$

where $k \geq 3$. Denote the $k \times k$ submatrix of $\frac{1}{2}\left(Q+Q^{t}\right)$ corresponding to $\mathcal{P}_{k}$ by $P_{k}$.

We may construct symmetric subpermutation matrices $A_{1}$ and $A_{2}$ similarly in Lemma 3.4 such that

$$
P_{k}=\frac{1}{2} A_{1}+\frac{1}{2} A_{2},
$$

and hence $\frac{1}{2}\left(Q+Q^{t}\right)$ is not an extreme point. 
Theorem 3.6. Let $Q$ be an $n \times n$ subpermutation matrix. Then $\frac{1}{2}\left(Q+Q^{t}\right)$ is an extreme point of $\omega_{n}^{t}$ if and only if each connected component of the loopy graph associated with $\frac{1}{2}\left(Q+Q^{t}\right)$ is one of the following:

(a) a loop

(b) an isolated vertex

(c) an independent edge (a path with length 1)

(d) an odd cycle.

Proof. Let $A=\frac{1}{2}\left(Q+Q^{t}\right)$. Since $Q$ is a subpermutation matrix, it contains at most one nonzero element in each row and each column, and hence $\frac{1}{2}\left(Q+Q^{t}\right)$ contains at most two nonzero elements in each row and each column which implies that the degree of each vertex in the associated loopy graph $G(A)$ is at most 2 . Therefore, each connected component of $G(A)$ must be one of the following:

(a) a loop

(b) a path longer than 1

(c) an isolated vertex

(d) an independent edge (a path with length 1)

(e) an odd cycle

(f) an even cycle.

Lemma 3.4 and Lemma 3.5 rule out the possibilities that $G(A)$ contains a connected component which is a path longer than 1 or an even cycle.

On the other hand, if each of connected component of $A$ is one of the four types listed in the theorem, then there exists a permutation matrix $P$ such that $P A P^{T}$ is a direct sum of some square matrices with smaller orders denoted by $A_{1}, A_{2}, \ldots, A_{k}$, and $G\left(A_{i}\right)$, the associated loopy graph of $A_{i}$, is one of these four types. It is suffice to show that each $A_{i}$ is extreme for $i=1,2, \ldots, k$.

If $G\left(A_{i}\right)$ is a loop, then $A_{i}$ is the $1 \times 1$ matrix $I_{1}$ which is extreme. If $G\left(A_{i}\right)$ is an isolated vertex, then $A_{i}$ is the $1 \times 1$ zero matrix. If $G\left(A_{i}\right)$ is an independent edge, then $A_{i}$ is the $2 \times 2$ matrix $\left(\begin{array}{ll}0 & 1 \\ 1 & 0\end{array}\right)$ which is a permutation matrix and hence extreme. If $G\left(A_{i}\right)$ is an odd cycle, then according to Theorem 3.1, each $A_{i}$ is an extreme point. Hence, $A$ is an extreme point.

\section{References}

[1] G. Birkhoff. Three observations on linear algebra. Univ. Nac. Tucumán. Revista A., 5:147-151, 1946.

[2] R.A. Brualdi, L. Cao. Symmetric, Hankel-symmetric, and centrosymmetric doubly stochastic matrices. Acta Math. Vietnam., 43(4):675-700, 2018.

[3] L. Cao, S. Koyuncu, T. Parme . A minimal completion of doubly substochastic matrix. Linear and Multilinear Algebra, 64(11):2313-2334, 2016.

[4] C.R. Johnson. Row stochastic matrices similar to doubly stochastic matrices. Linear and Multilinear Algebra, 10(2):113-130, 1981.

[5] M. Katz. On the extreme points of a certain convex polytope. J. Combin. Theory, 8:417-423, 1970.

[6] L. Mirsky. On a convex set of matrices. Arch. Math., 10:88-92, 1959. 